\title{
Study Regarding the Perception of Small Farmers from the South- East of Romania on Social Agricultural Cooperatives
}

\author{
By Virlanuta Florina Oana ${ }^{1}$, Moisescu Florentina ${ }^{1}$, Manea Daniela Ludmila ${ }^{1}$
}

\begin{abstract}
Social economy suggests new, innovative solutions to certain social, economic or environmental problems of communities in order to meet the needs expressed by community members, which are insufficiently covered or ignored by the public or the private sector. The domain represents a priority both at the national and at the European level for the period $2014-2020$, being a research topic for the academic environment and the subject of a great number of scientific conferences and research studies. The study below presents the original research performed by the authors in order to determine the social agricultural cooperative acceptability degree among the farmers from the south-east of Romania. The first stage of the research strategy materialised in various documentation activities regarding the draw-up of a questionnaire and finished with the completion of the questionnaire and of the computer-based methodology used for its processing. The second stage involved the design of the sample structure and size and the processing methodology. In the third stage, we put forward the working hypotheses and we identified the connection degree between the variables that constituted the basis for making the hypotheses.
\end{abstract}

Keywords: social economy, social agricultural cooperative, sustainable development

\section{Introduction}

Social economy suggests new, innovative solutions to certain social, economic or environmental problems of communities in order to meet the needs expressed by community members, which are insufficiently covered or ignored by the public or the private sector. (Arpinte, Baboi, Cace, Tomescu, \& Stanescu, 2010) The domain represents a priority both at the national and at the European level for the period 2014 2020, being a research topic for the academic environment and the subject of a great number of scientific conferences and research studies. According to the recommendations made by the European Commission (CIRIEC, 2015), the economic and social models must be rethought and adapted to the present context, and social entities must support social innovation as well as an inclusive and durable economic growth. Sustainable development is a general and fundamental objective of the European Union aimed at continuous improvement of the quality of life, based on interconnections among economic development, environmental protection and social justice. (Huttmanova, 2016)

The legislative initiatives regarding the freedom of association led to the development of social economy throughout history. Thus, the freedom of association was acknowledged even from the $19^{\text {th }}$ century in Great Britain, Germany, the Netherlands, France and In the modern period, in 1980, the representatives of the mutual and associative 
cooperative sectors drew up the Social Economy Charter, which was updated in 1995, and which reiterated the values and organisation method of social economy by highlighting the fundamental principles of this sector: solidarity, responsibility, freedom, equal chances for all the members of the organisation, and mutual respect. In sustainable agriculture and rural development, a special place is occupied by family farms, which, despite the pressure of agri-industrial corporations, still are a basic organisational form of agriculture, being a socially attractive way of agricultural production. (Wrzaszcz \& Zegar, 2016)

The objectives of social economy regarding employment, the development of social services and the consolidation of social cohesion are found in the creation and development of the social economy entities from the two sub-sectors: commercial and non-commercial. (Longo, Clark, Shriver, \& Clausen, 2015) The major orientations regarding public policies refer to social economy by means of social inclusion policies, of workforce market insertion policies, of local development policies and job creation policies. (Research Report Regarding Social Economy in Romania from a Comparative European Perspective, 2015)

In Romania, according to (Stanescu, 2013), the social economy sector is in an early stage of development, being undersized as far as the number of employees is concerned as compared to its percentage in other European countries. The research performed by Stanescu is also confirmed by (Barna, 2014), who proves that, in 2013, the social economy sector in Romania included 39,347 active organisations, which hold fixed assets of 13,917,508 thousand lei, revenues amounting to 12,298,111 thousand lei, and which hired a number of approximately 131,127 people (Barna, 2014).

\section{The Stages of the Research and the Methods Used}

The study below presents the original research performed by the authors in order to determine the social agricultural cooperative acceptability degree among the farmers from the south-east of Romania.

The first stage of the research strategy materialised in various documentation activities regarding the draw-up of a questionnaire and finished with the completion of the questionnaire and of the computer-based methodology used for its processing. The total number of items used in the questionnaire was of 14, out of which the first 11 items focused on the fundamental problems of the research (the human resources management policy, the affiliation to a certain county, the respondents' expectations regarding the evolution of social agricultural cooperatives, their activity sector, the social responsibility area, the acceptability degree of the social agricultural cooperatives, the development of local communities), whereas the last 3 questions were dedicated to gathering information on the respondent profile (gender, age, activity sector).

The second stage involved the design of the sample structure and size and the processing methodology. Filling in the questionnaire was voluntary, an agreement questionnaire being used. The results are relevant due to the fact that only the people who were interested in the topic expressed their opinions. The maximum permissible error was determined by using formula (1), whereas the size of the representative sample was determined by means of formula (2). 
The sample size to be investigated was designed by taking into account the fact that the maximum permissible limit error in the case of this type of survey is:

$$
\Delta_{\bar{x}}=z_{\alpha} \frac{\sigma}{\sqrt{n}} \sqrt{1-\frac{n}{N}}
$$

From where $n$ - the size of the sample is extracted:

$n=\frac{Z^{2} \sigma^{2}}{\Delta \frac{2}{x}+\frac{Z^{2} \sigma^{2}}{N}}$

where:

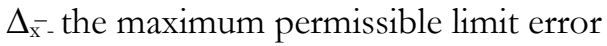

$\sigma-$ the standard deviation

$\mathrm{Z}_{\alpha}$ - the value of the Laplace variable for the $\alpha$ risk

$\alpha$ - the value of the $1^{\text {st }}$ degree risk

$\mathrm{P}=1-\alpha$ the reliability probability

$\mathrm{N}$ - the number of the general population

$\mathrm{n}$ - the size of the investigated sample

- The sufficiently reliable, close to certainty, result guarantee probability $(\mathrm{P}=1-\alpha)$, from a practical point of view;

- The dispersion of the feature in the general population $\sigma^{2}$, or in its estimator;

The values resulted from the calculation are increased both for obtaining a whole number and out of practical considerations so that the research could not be endangered, taking into consideration the fact that a certain number of questionnaires may be rejected. After establishing the size of the sample, we encoded the answers in order to facilitate the subsequent processing. After gathering, checking and validating the questionnaires, the obtained data were entered in SPSS.

In the third stage, we put forward the working hypotheses and we identified the connection degree between the variables that constituted the basis for making the hypotheses.

\section{The Results of the Investigation}

\subsection{The respondent sample characterisation}

Table no 1. The respondent sample characterisation divided into the counties where they operate

\begin{tabular}{|c|c|c|c|c|}
\hline City & $\begin{array}{c}\text { Number of } \\
\text { respondents }\end{array}$ & $\begin{array}{c}\text { Pondere } \\
(\%)\end{array}$ & $\begin{array}{c}\text { Brăila } \\
19 \%\end{array}$ & \begin{tabular}{|l} 
Galați \\
$22 \%$
\end{tabular} \\
\hline 1. Galaţi & 69 & 21,97 & \multirow{4}{*}{$\begin{array}{c}\text { Constanța } \\
14 \%\end{array}$} & \multirow{4}{*}{$\begin{array}{c}\text { Vrancea } \\
16 \%\end{array}$} \\
\hline 2. Vrancea & 52 & 16,56 & & \\
\hline 3. Buzău & 49 & 15,60 & & \\
\hline 4. Tulcea & 42 & 13,38 & & \\
\hline 5. Constanța & 43 & 13,70 & \multirow{3}{*}{$\begin{array}{c}\text { Tulcea } \\
13 \%\end{array}$} & \\
\hline 6. Brăila & 59 & 18,79 & & \multirow{2}{*}{$\begin{array}{c}\text { Buzău } \\
16 \%\end{array}$} \\
\hline Total & 314 & 100 & & \\
\hline
\end{tabular}

Source: Authors' own research 
Table no 2 . The sample structure by the respondents' activity sector

\begin{tabular}{|c|c|c|c|c|c|}
\hline Activity sector & $\begin{array}{c}\text { Number of } \\
\text { respondents }\end{array}$ & $\begin{array}{c}\text { Pondere } \\
(\%)\end{array}$ & \multirow[t]{2}{*}{$\begin{array}{c}\text { Zootehnie } \\
17 \%\end{array}$} & \multicolumn{2}{|l|}{$\begin{array}{c}\text { Agroturis } \\
\text { m } \\
8 \%\end{array}$} \\
\hline Viticulture & 24 & 7,64 & & \multirow{2}{*}{\multicolumn{2}{|c|}{\begin{tabular}{|c|c} 
Viticultură \\
$8 \%$
\end{tabular}}} \\
\hline Fruits and vegetables & 145 & 46,17 & \multirow[b]{5}{*}{$\begin{array}{c}\text { Apicultură } \\
21 \%\end{array}$} & & \\
\hline Apiculture & 66 & 21,03 & & & \\
\hline Zootechnics & 54 & 17,20 & & & \\
\hline Agro tourism & 25 & 7,96 & & \multirow{2}{*}{\multicolumn{2}{|c|}{$\begin{array}{c}\begin{array}{c}\text { Fructe și } \\
\text { legume } \\
46 \%\end{array} \\
\end{array}$}} \\
\hline Total & 314 & 100,0 & & & \\
\hline
\end{tabular}

Source: Authors' own research

Table no 3. The sample structure by age

\begin{tabular}{|c|c|c|c|c|c|}
\hline Age & $\begin{array}{c}\text { Number of } \\
\text { respondents }\end{array}$ & $\begin{array}{c}\text { Pondere } \\
\mathbf{( \% )}\end{array}$ & $\begin{array}{c}>45 \\
12 \%\end{array}$ & $\begin{array}{c}18-25 \\
13 \%\end{array}$ \\
\hline $18-25$ & 41 & 13,05 & & \\
\hline $25-35$ & 123 & 39,14 & & \\
\hline $35-45$ & 112 & 35,67 & & \\
\hline$>45$ & 38 & 12,01 & & \\
\hline Total & 314 & $\mathbf{1 0 0 , 0}$ & $35-45$ & $36 \%$ \\
\hline
\end{tabular}

Source: Authors' own research

The collected data were processed by means of the SPSS programme. Four working hypotheses were made, $\mathrm{H} 1-\mathrm{H} 4$, which took into consideration eight variables (the age of the respondents, the human resources management policy, the affiliation to a certain county, their expectations regarding the evolution of the social agricultural cooperatives, the activity sector, the social responsibility area, the acceptability degree of the social agricultural cooperatives, the development of local communities).

H1: The age of the respondents significantly influences the adopted human resources management policy regarding the employment of socially vulnerable people.

H2: The affiliation to a certain county from the south-east region, in which the respondents operate, significantly influences their expectations in connection with the evolution of social agricultural cooperatives.

H3: The activity sector in which the respondents operate significantly influences the approach of a certain social responsibility area.

H4: The acceptability degree of the social agricultural cooperatives in Romania significantly influences the development of local communities

\subsection{The results regarding the verification of the proposed working hypotheses}

The verification of the results' validity was performed by means of the Pearson Test. 
Table no 4. The verification of $\mathrm{H} 1$ (The age of the respondents significantly influences the adopted human resources management policy regarding the employment of socially vulnerable people)

\begin{tabular}{|c|c|c|c|c|c|c|c|c|}
\hline \multicolumn{9}{|c|}{ Respondents Age * Employing socially vulnerable people Crosstabulation } \\
\hline & & & \multicolumn{5}{|c|}{ Employing socially vulnerable people } & \multirow[b]{2}{*}{ Total } \\
\hline & & & $\begin{array}{l}\text { Agree in a very } \\
\text { large mesure }\end{array}$ & $\begin{array}{c}\text { Agree in a } \\
\text { large mesure }\end{array}$ & Undecided & $\begin{array}{l}\text { Agree on a } \\
\text { small scale }\end{array}$ & $\begin{array}{c}\text { Agree on a very } \\
\text { small scale }\end{array}$ & \\
\hline \multirow[t]{8}{*}{ Respondents Age } & \multirow[t]{2}{*}{ 18-25 years } & Count & 6 & 20 & $6 \mathrm{a}$ & $9 \mathrm{a}$ & $\mathrm{O}_{\mathrm{a}}$ & 41 \\
\hline & & $\begin{array}{l}\% \text { within Employing socially } \\
\text { vulnerable people }\end{array}$ & $13,0 \%$ & $11,9 \%$ & $13,3 \%$ & $18,8 \%$ & $0,0 \%$ & $13,1 \%$ \\
\hline & \multirow[t]{2}{*}{$26-35$ years } & Count & $21_{\mathrm{a}}$ & $70_{\mathrm{a}}$ & $15_{\mathrm{a}}$ & $17 \mathrm{a}$ & $0_{\mathrm{a}}$ & 123 \\
\hline & & $\begin{array}{l}\% \text { within Employing socially } \\
\text { vulnerable people }\end{array}$ & $45,7 \%$ & $41,7 \%$ & $33,3 \%$ & $35,4 \%$ & $0,0 \%$ & $39,2 \%$ \\
\hline & \multirow[t]{2}{*}{$36-45$ years } & Count & 15 & 54 & $21_{\mathrm{a}}$ & $17_{\mathrm{a}}$ & $5 a$ & 112 \\
\hline & & $\begin{array}{l}\% \text { within Employing socially } \\
\text { vulnerable people }\end{array}$ & $32,6 \%$ & $32,1 \%$ & $46,7 \%$ & $35,4 \%$ & $71,4 \%$ & $35,7 \%$ \\
\hline & \multirow[t]{2}{*}{$>45$ years } & Count & 4 & $24 \mathrm{a}$ & $3 a$ & $5 \mathrm{a}$ & $2 \mathrm{a}$ & 38 \\
\hline & & $\begin{array}{l}\% \text { within Employing socially } \\
\text { vulnerable people }\end{array}$ & $8,7 \%$ & $14,3 \%$ & $6,7 \%$ & $10,4 \%$ & $28,6 \%$ & $12,1 \%$ \\
\hline \multirow{2}{*}{\multicolumn{2}{|c|}{ Total }} & Count & 46 & 168 & 45 & 48 & 7 & 314 \\
\hline & & $\begin{array}{l}\% \text { within Employing socially } \\
\text { vulnerable people }\end{array}$ & $100,0 \%$ & $100,0 \%$ & $100,0 \%$ & $100,0 \%$ & $100,0 \%$ & $100,0 \%$ \\
\hline
\end{tabular}

Source: Authors' own research

Chi-Square Tests

\begin{tabular}{|c|c|c|c|}
\hline & Value & df & Asymp. Sig. (2-sided) \\
\hline Pearson Chi-Square & $14,971^{\text {a }}$ & 12 &, 243 \\
Likelihood Ratio & 17,596 & 12 &, 129 \\
Linear-by-Linear Association &, 704 & 1 &, 401 \\
N of Valid Cases & 314 & & \\
\hline
\end{tabular}

a. 4 cells $(20,0 \%)$ have expected count less than 5 . The minimum expected count is, 85 .

Chi-Square Distribution Table

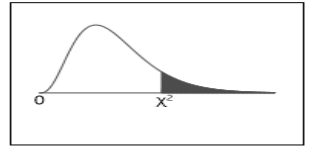

The shaded area is equal to $\alpha$ for $x^{2}=x_{x}^{2}$

\begin{tabular}{|c|c|c|c|c|c|c|c|c|c|c|}
\hline$d f$ & $x_{995}^{2}$ & $x^{2}=000$ & $x_{275}^{2}$ & $x_{950}^{2}$ & $x^{2}$ & $x_{1100}^{2}$ & $x^{2} 050$ & $x_{025}^{2}$ & $x_{-010}^{2}$ & $x_{005}^{2}$ \\
\hline (11 & 0.000 & 0.000 & 0.001 & 0.004 & 0.016 & 2.706 & 3.841 & 5.024 & 6.635 & 7.879 \\
\hline 2 & 0.010 & 0.020 & 0.051 & 0.103 & 0.211 & 4.605 & 5.991 & $7-378$ & 9.210 & 10.597 \\
\hline 3 & 0.072 & 0.115 & 0.216 & 0.352 & 0.584 & 6.251 & 7.815 & $9-348$ & 11.345 & 12.838 \\
\hline 4 & 0.207 & 0.297 & 0.484 & 0.711 & 1.064 & 7.779 & 9.488 & 11.143 & 13.277 & 14.860 \\
\hline 5 & 0.412 & 0.554 & 0.831 & 1.145 & 1.610 & 9.236 & 11.070 & 12.833 & 15.086 & 16.750 \\
\hline 6 & 0.676 & 0.872 & 1.237 & 1.635 & 2.204 & 10.645 & 12.592 & 14.449 & 16.812 & 18.548 \\
\hline 7 & 0.989 & 1.239 & 1.690 & 2.167 & 2.833 & 12.017 & 14.067 & 16.013 & 18.475 & 20.278 \\
\hline 8 & 1.344 & 1.646 & 2.180 & 2.733 & 3.490 & 13.362 & 15.507 & 17.535 & 20.090 & 21.955 \\
\hline 9 & $\begin{array}{l}.374 \\
1.7355\end{array}$ & $\begin{array}{l}1.0700 \\
2.088\end{array}$ & $\begin{array}{l}2.7000 \\
2.700\end{array}$ & $\begin{array}{l}2.3 .35 \\
3.325\end{array}$ & 4.168 & $\begin{array}{l}13.362 \\
14.684\end{array}$ & 16.919 & 19.023 & 21.6666 & $\begin{array}{l}2.353 \\
23.589\end{array}$ \\
\hline 10 & $\begin{array}{l}1.1350 \\
2.156\end{array}$ & $\begin{array}{l}2.008 \\
2.558\end{array}$ & 3.247 & $\begin{array}{l}3.380 \\
3.940\end{array}$ & $\begin{array}{l}4.865 \\
4.865\end{array}$ & $\begin{array}{l}15.987\end{array}$ & $\begin{array}{l}18.307 \\
18.307\end{array}$ & $\begin{array}{l}19023 \\
20.483\end{array}$ & $\begin{array}{l}23.200 \\
23.209\end{array}$ & $\begin{array}{l}25.0898 \\
25.188\end{array}$ \\
\hline 11 & 2.603 & 3.053 & 3.816 & 4.575 & 5.578 & 17.275 & 19.675 & 21.920 & 24.725 & 26.757 \\
\hline 12 & 3.074 & 3.571 & 4.404 & 5.226 & 6.304 & 18.549 & 21.026 & 23.357 & 26.217 & 28.300 \\
\hline 13 & 3.565 & 4.107 & 5.009 & 5.892 & 7.042 & 19.812 & 22.362 & 24.736 & 27.688 & 29.819 \\
\hline 11 & 1.075 & 4.660 & 5.629 & 6.571 & 7.700 & 21.061 & 23.685 & 26.119 & 29.1 .11 & 31.310 \\
\hline 15 & 4.601 & 5.229 & 6.262 & 7.261 & 8.547 & 22.307 & 24.996 & 27.488 & 30.5 .78 & 32.801 \\
\hline 16 & 5.142 & 5.812 & 6.908 & 7.962 & 9.312 & 23.542 & 26.296 & 28.815 & 32.000 & 34.267 \\
\hline 17 & 5.697 & 6.408 & 7.564 & 8.672 & 10.085 & 24.769 & 27.587 & 30.191 & 33.409 & 35.718 \\
\hline 18 & 6.2665 & 7.015 & 8.231 & 9.390 & 10.865 & 25.989 & 28.860 & 31.526 & 34.805 & 37.156 \\
\hline 19 & 6.844 & 7.633 & 8.907 & 10.117 & 11.65 .1 & 27.204 & 30.144 & 32.852 & 36.191 & 38.582 \\
\hline 20 & 7.434 & 8.260 & 9.591 & 10.851 & 12. 443 & 28.412 & 31.410 & 34.170 & 37.566 & 39.997 \\
\hline 21 & 8.034 & 8.897 & 10.283 & 11.591 & 13.240 & 29.615 & 32.671 & 35.479 & 38.932 & 41.401 \\
\hline 22 & 8.643 & 9.542 & 10.982 & 12.338 & 14.041 & 30.813 & 33.924 & 36.781 & 40.289 & 42.796 \\
\hline 23 & 9.2600 & 10.196 & 11.689 & 13.091 & 14.848 & 32.007 & 35.172 & 38.076 & 41.6388 & 44.181 \\
\hline 24 & 9.886 & 10.856 & 12.401 & 13.848 & 15.659 & 33.196 & 36.415 & 39.364 & 42.980 & 45.559 \\
\hline 25 & $\begin{array}{l}3.080 \\
10.520\end{array}$ & $\begin{array}{l}11.524 \\
11.524\end{array}$ & $\begin{array}{l}13.120 \\
\end{array}$ & 14.611 & $\begin{array}{l}15.0393 \\
16.473\end{array}$ & 34.382 & $\begin{array}{r}30.452 \\
37.652\end{array}$ & 40.646 & 44.314 & $\begin{array}{r}46.928 \\
46.928\end{array}$ \\
\hline 26 & 11.160 & 12.198 & 13.814 & 15.379 & 17.292 & 35.563 & 38.885 & 41.923 & 45.642 & 48.290 \\
\hline 27 & 11.808 & 12.879 & 14.573 & 16.151 & 18.114 & 36.741 & 40.113 & 43.195 & 46.963 & 49.645 \\
\hline 28 & 12.461 & 13.565 & 15.308 & 16.928 & 18.939 & 37.916 & 41.337 & 44.46i & 48.278 & 50.993 \\
\hline 29 & 13.121 & 14.256 & 16.047 & 17.708 & 19.768 & 39.087 & 42.557 & 45.722 & 49.588 & 52.336 \\
\hline
\end{tabular}


The analysis of the data leads to a $\mathrm{Pv}_{\mathrm{v}}$ asymptotic significance value of 0.243 , which exceeds the maximum permissible limit of 0.05 . The determined value of the Pearson Chi-Square coefficient (14.971) is smaller than the $\mathrm{df}$ limit value (21.026). According to these results, the hypothesis $\mathrm{H} 1$ is invalidated. In the analysed areas, a correlation between the age of the respondents and the employment of the socially vulnerable people can't be established.

Table no 5. The verification of H2 (The affiliation to a certain county from the south-east region, in which the respondents operate, significantly influences their expectations in connection with the evolution of social agricultural cooperatives)

County * Expectations Cross tabulation

\begin{tabular}{|c|c|c|c|c|c|c|}
\hline & \multicolumn{3}{|c|}{ Expectations } & \multirow{2}{*}{ Total } \\
\hline & & & Legislative stability & Fiscal facilities & Subsidies & \\
\hline \multirow{14}{*}{ City } & \multirow{2}{*}{ Galati } & Count & $11_{\mathrm{a}}$ & $22_{\mathrm{a}}$ & $36_{a}$ & 69 \\
\hline & & $\%$ within Expectations & $26,8 \%$ & $22,4 \%$ & $20,6 \%$ & $22,0 \%$ \\
\hline & \multirow{2}{*}{ Vrancea } & Count & $6_{a}$ & $19 \mathrm{a}$ & $27 \mathrm{a}$ & 52 \\
\hline & & $\%$ within Expectations & $14,6 \%$ & $19,4 \%$ & $15,4 \%$ & $16,6 \%$ \\
\hline & \multirow{2}{*}{ Buzau } & Count & $4 a$ & $14_{a}$ & $31_{a}$ & 49 \\
\hline & & $\%$ within Expectations & $9,8 \%$ & $14,3 \%$ & $17,7 \%$ & $15,6 \%$ \\
\hline & \multirow{2}{*}{ Tulcea } & Count & $6 a$ & $9 \mathrm{a}$ & $27 \mathrm{a}$ & 42 \\
\hline & & $\%$ within Expectations & $14,6 \%$ & $9,2 \%$ & $15,4 \%$ & $13,4 \%$ \\
\hline & \multirow{2}{*}{ Constanta } & Count & $5 a$ & $16 a$ & $22_{a}$ & 43 \\
\hline & & $\%$ within Expectations & $12,2 \%$ & $16,3 \%$ & $12,6 \%$ & $13,7 \%$ \\
\hline & \multirow{2}{*}{ Braila } & Count & $9 \mathrm{a}$ & $18 \mathrm{a}$ & $32 \mathrm{a}$ & 59 \\
\hline & & $\%$ within Expectations & $22,0 \%$ & $18,4 \%$ & $18,3 \%$ & $18,8 \%$ \\
\hline & \multirow{2}{*}{ Total } & Count & 41 & 98 & 175 & 314 \\
\hline & & $\%$ within Expectations & $100,0 \%$ & $100,0 \%$ & $100,0 \%$ & $100,0 \%$ \\
\hline
\end{tabular}

Each subscript letter denotes a subset of Expectations categories whose column proportions do not differ

Source: Authors' own research significantly from each other at the ,05 level.

Chi-Square Tests

\begin{tabular}{|c|c|c|c|}
\hline & Value & df & Asymp. Sig. (2-sided) \\
\hline Pearson Chi-Square & $5,677^{\mathrm{a}}$ & 10 &, 842 \\
Likelihood Ratio & 5,855 & 10 &, 827 \\
Linear-by-Linear Association &, 029 & 1 &, 864 \\
N of Valid Cases & 314 & & \\
\hline
\end{tabular}

a. 0 cells $(, 0 \%)$ have expected count less than 5 . The minimum expected count is 5,48 .

According to these results, there isn't any connection between the two variables because the $\mathrm{Pv}$ asymptotic significance value $(0.842)$ is bigger than the permissible significance value of 0.05 , and the Pearson Chi-Square value (5.677) is smaller than the df value (18.307). 
Chi-Square Distribution Table

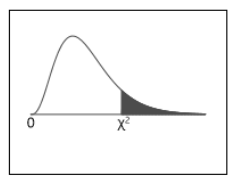

The shaded area is equal to $\alpha$ for $\chi^{2}=\chi_{\alpha}^{2}$.

\begin{tabular}{|c|c|c|c|c|c|c|c|c|c|c|}
\hline$d f$ & $\chi_{995}^{2}$ & $\chi_{990}^{2}$ & $\chi_{9975}^{2}$ & $\chi_{950}^{2}$ & $\chi_{990}^{2}$ & $\chi_{1100}^{2}$ & $\chi_{.050}^{2}$ & $\chi_{.025}^{2}$ & $\chi_{.010}^{2}$ & $\chi_{.005}^{2}$ \\
\hline 1 & 0.000 & 0.000 & 0.001 & 0.004 & 0.016 & 2.706 & 3.841 & $\overline{5.024}$ & 6.635 & 27.879 \\
\hline 2 & 0.010 & 0.020 & 0.051 & 0.103 & 0.211 & 4.605 & 5.991 & 7.378 & 9.210 & 10.597 \\
\hline 3 & 0.072 & 0.115 & 0.216 & 0.352 & 0.584 & 6.251 & 7.815 & 9.348 & 11.345 & 12.838 \\
\hline 4 & 0.207 & 0.297 & 0.484 & 0.711 & 1.064 & 7.779 & 9.488 & 11.143 & 13.277 & 14.860 \\
\hline 5 & 0.412 & 0.554 & 0.831 & 1.145 & 1.610 & 9.236 & 11.070 & 12.833 & 15.086 & 16.750 \\
\hline 6 & 0.676 & 0.872 & 1.237 & 1.635 & 2.204 & 10.645 & 12.592 & 14.449 & 16.812 & 18.548 \\
\hline 7 & 0.989 & 1.239 & 1.690 & 2.167 & 2.833 & 12.017 & 14.067 & 16.013 & 18.475 & 20.278 \\
\hline 8 & 1.344 & 1.646 & 2.180 & 2.733 & 3.490 & 13.362 & 15.507 & 17.535 & 20.090 & 21.955 \\
\hline 9 & 1.735 & 2.088 & 2.700 & 3.325 & 4.168 & 14.684 & 16.919 & 19.023 & 21.666 & 23.589 \\
\hline 10 & 2.156 & 2.558 & 3.247 & 3.940 & 4.865 & 15.987 & 18.307 & 20.483 & 23.209 & 25.188 \\
\hline 11 & 2.603 & 3.053 & 3.816 & 4.575 & 5.578 & 17.275 & 19.675 & 21.920 & 24.725 & 26.757 \\
\hline 12 & 3.074 & 3.571 & 4.404 & 5.226 & 6.304 & 18.549 & 21.026 & 23.337 & 26.217 & 28.300 \\
\hline 13 & 3.565 & 4.107 & 5.009 & 5.892 & 7.042 & 19.812 & 22.362 & 24.736 & 27.688 & 29.819 \\
\hline 14 & 4.075 & 4.660 & 5.629 & 6.571 & 7.790 & 21.064 & 23.685 & 26.119 & 29.141 & 31.319 \\
\hline 15 & 4.601 & 5.229 & 6.262 & 7.261 & 8.547 & 22.307 & 24.996 & 27.488 & 30.578 & 32.801 \\
\hline 16 & 5.142 & 5,812 & 6.908 & 7.962 & 9.312 & 23.542 & 26.296 & 28.845 & 32.000 & 34.267 \\
\hline 17 & 5.697 & 6.408 & 7.564 & 8.672 & 10.085 & 24.769 & 27.587 & 30.191 & 33.409 & 35.718 \\
\hline 18 & 6.265 & 7.015 & 8.231 & 9.390 & 10.865 & 25.989 & 28.869 & 31.526 & 34.805 & 37.156 \\
\hline 19 & 6.844 & 7.633 & 8.907 & 10.117 & 11.651 & 27.204 & 30.144 & 32.852 & 36.191 & 38.582 \\
\hline 20 & 7.434 & 8.260 & 9.591 & 10.851 & 12.443 & 28.412 & 31.410 & $\begin{array}{l}34.170 \\
3\end{array}$ & 37.566 & 39.997 \\
\hline 21 & 8.034 & 8.897 & 10.283 & 11.591 & 13.240 & 29.615 & 32.671 & 35.479 & 38.932 & 41.401 \\
\hline 22 & 8.643 & 9.542 & 10.982 & 12.338 & 14.041 & 30.813 & 33.924 & 36.781 & 40.289 & 42.796 \\
\hline 23 & 9.260 & 10.196 & 11.689 & 13.091 & 14.848 & 32.007 & 35.172 & 38.076 & 41.638 & 44.181 \\
\hline 24 & 9.886 & 10.856 & 12.401 & 13.848 & $\begin{array}{l}15.659 \\
\end{array}$ & 33.196 & 36.415 & 39.364 & 42.980 & 45.559 \\
\hline 25 & 10.520 & 11.524 & 13.120 & 14.611 & 16.473 & 34.382 & 37.652 & 40.646 & 44.314 & 46.928 \\
\hline 26 & 11.160 & 12.198 & 13.844 & 15.379 & 17.292 & 35.563 & 38.885 & 41.923 & 45.642 & 48.290 \\
\hline 27 & 11.808 & 12.879 & 14.573 & 16.1 & 18.114 & 36.741 & 40.113 & 43.195 & 46.963 & 49.645 \\
\hline 28 & $\begin{array}{l}12.461 \\
\end{array}$ & 13.565 & $\begin{array}{l}15.308 \\
\end{array}$ & $\begin{array}{l}16.928 \\
\end{array}$ & 18.939 & $\begin{array}{l}37.916 \\
3717\end{array}$ & $\begin{array}{l}41.337 \\
4\end{array}$ & $\begin{array}{l}4.153 \\
44.461\end{array}$ & 48.278 & 50.993 \\
\hline 29 & 13.121 & 14.256 & 16.047 & 17.708 & 19.768 & 39.087 & 42.557 & 45.722 & 49.588 & 52.336 \\
\hline
\end{tabular}

Therefore, we may draw the conclusion that the hypothesis is invalidated and that there isn't any connection between the two variables (the affiliation to a certain county from the south-east region, in which the respondents operate, and their expectations regarding the evolution of the social agricultural cooperatives).

Table no 6. The verification of $\mathrm{H} 3$ (The activity sector in which the respondents operate significantly influences the approach of a certain social responsibility area)

Activity sector * Responsability area Crosstabulation

\begin{tabular}{|c|c|c|c|c|c|c|c|}
\hline & & & \multicolumn{4}{|c|}{ Responsability area } & \multirow[b]{2}{*}{ Total } \\
\hline & & & $\begin{array}{c}\text { Environment } \\
\text { protection }\end{array}$ & $\begin{array}{l}\text { Employees } \\
\text { protection }\end{array}$ & $\begin{array}{l}\text { Education } \\
\text { promotion }\end{array}$ & $\begin{array}{c}\text { Culture } \\
\text { promoting }\end{array}$ & \\
\hline \multirow[t]{10}{*}{ Activity sector } & \multirow[t]{2}{*}{ Viticulture } & Count & $16_{a, b}$ & $2_{a, b}$ & $5_{b}$ & 1 & 24 \\
\hline & & $\%$ within Responsability area & $8,0 \%$ & $5,9 \%$ & $19,2 \%$ & $1,9 \%$ & $7,6 \%$ \\
\hline & \multirow{2}{*}{$\begin{array}{l}\text { Fruits and } \\
\text { vegetables }\end{array}$} & Count & $94 \mathrm{a}$ & 14 a & $9_{\mathrm{a}}$ & 28 & 145 \\
\hline & & $\%$ within Responsability area & $47,0 \%$ & $41,2 \%$ & $34,6 \%$ & $51,9 \%$ & $46,2 \%$ \\
\hline & \multirow[t]{2}{*}{ Apiculture } & Count & 39 & $9_{\mathrm{a}}$ & $4 a$ & 14 & 66 \\
\hline & & $\%$ within Responsability area & $19,5 \%$ & $26,5 \%$ & $15,4 \%$ & $25,9 \%$ & $21,0 \%$ \\
\hline & \multirow[t]{2}{*}{ Zootechnics } & Count & $38_{a}$ & 6 & $4 a$ & 6 & 54 \\
\hline & & $\%$ within Responsability area & $19,0 \%$ & $17,6 \%$ & $15,4 \%$ & $11,1 \%$ & $17,2 \%$ \\
\hline & \multirow[t]{2}{*}{ Agrotourism } & Count & $13 \mathrm{a}$ & $3 a$ & $4 a$ & 5 & 25 \\
\hline & & $\%$ within Responsability area & $6,5 \%$ & $8,8 \%$ & $15,4 \%$ & $9,3 \%$ & $8,0 \%$ \\
\hline \multirow{2}{*}{\multicolumn{2}{|c|}{ Total }} & Count & 200 & 34 & 26 & 54 & 314 \\
\hline & & $\%$ within Responsability area & $100,0 \%$ & $100,0 \%$ & $100,0 \%$ & $100,0 \%$ & $100,0 \%$ \\
\hline
\end{tabular}

Each subscript letter denotes a subset of Responsability area categories whose column proportions do not differ significantly from each other at the, 05 level.

Source: Authors' own research

(C) 2017 The Authors. Journal Compilation ～(C) 2017 European Center of Sustainable Development. 


\section{Chi-Square Tests}

\begin{tabular}{|c|c|c|c|}
\hline & Value & df & Asymp. Sig. (2-sided) \\
\hline Pearson Chi-Square & $14,239^{\mathrm{a}}$ & 12 &, 286 \\
Likelihood Ratio & 13,763 & 12 &, 316 \\
Linear-by-Linear Association &, 136 & 1 &, 712 \\
N of Valid Cases & 314 & & \\
\hline
\end{tabular}

a. 7 cells $(35,0 \%)$ have expected count less than 5 . The minimum expected count is 1,99 .

According to these results, there isn't any connection between the two variables because the $\mathrm{Pv}$ asymptotic significance value $(0.286)$ is bigger than the permissible significance value of 0.05 , and the Pearson Chi-Square value (14.239) is smaller than the df value (21.026).

Therefore, we may draw the conclusion that the hypothesis is invalidated and that there isn't any connection between the two variables (the activity sector in which the respondents operate and the approach of a certain area of social responsibility).

Table no 7. The verification of $\mathrm{H} 4$ (The acceptability degree of the social agricultural cooperatives in Romania significantly influences the development of local communities)

\begin{tabular}{|c|c|c|c|c|c|c|c|c|}
\hline \multicolumn{9}{|c|}{ Acceptability degree of social agricultural cooperatives ${ }^{*}$ The influence of social agricultural cooperatives to local development Crosstabulation } \\
\hline & & & \multicolumn{5}{|c|}{ The influence of social agricultural cooperatives to local development } & \multirow[b]{2}{*}{ Total } \\
\hline & & & Veryhigh & High & Medium & Lower & Very lower & \\
\hline \multirow{8}{*}{$\begin{array}{l}\text { Acceptability degree of social } \\
\text { agricultural cooperatives }\end{array}$} & \multirow[t]{2}{*}{ Ridicat } & Count & $3 a$ & $77_{\mathrm{a}}$ & $0_{b}$ & $0_{b}$ & $\mathrm{O}_{b}$ & 80 \\
\hline & & $\begin{array}{l}\% \text { within The influence of social agricultural } \\
\text { cooperatives to local development }\end{array}$ & $100,0 \%$ & $64,2 \%$ & $0,0 \%$ & $0,0 \%$ & $0,0 \%$ & $25,5 \%$ \\
\hline & \multirow[t]{2}{*}{ Mediu } & Count & $0_{a, b, c, d, e}$ & $22_{\mathrm{d}, \mathrm{e}}$ & $31_{c}$ & $26_{b, e}$ & $0_{a, d}$ & 79 \\
\hline & & $\begin{array}{l}\% \text { within The influence of social agricultural } \\
\text { cooperatives to local development }\end{array}$ & $0,0 \%$ & $18,3 \%$ & $48,4 \%$ & $26,3 \%$ & $0,0 \%$ & $25,2 \%$ \\
\hline & \multirow[t]{2}{*}{ Scazut } & Count & $0_{a, b}$ & $21_{b}$ & $31_{\mathrm{a}}$ & $60_{\mathrm{a}}$ & $13_{a}$ & 125 \\
\hline & & $\begin{array}{l}\% \text { within The influence of social agricultural } \\
\text { cooperatives to local development }\end{array}$ & $0,0 \%$ & $17,5 \%$ & $48,4 \%$ & $60,6 \%$ & $46,4 \%$ & $39,8 \%$ \\
\hline & \multirow{2}{*}{$\begin{array}{l}\text { Foarte } \\
\text { scazut }\end{array}$} & Count & $0_{a, b, c}$ & $0_{c}$ & $22_{b, c}$ & $13 \mathrm{~b}$ & $15 \mathrm{a}$ & 30 \\
\hline & & $\begin{array}{l}\% \text { within The influence of social agricultural } \\
\text { cooperatives to local development }\end{array}$ & $0,0 \%$ & $0,0 \%$ & $3,1 \%$ & $13,1 \%$ & $53,6 \%$ & $9,6 \%$ \\
\hline \multirow{2}{*}{\multicolumn{2}{|c|}{ Total }} & Count & 3 & 120 & 64 & 99 & 28 & 314 \\
\hline & & $\begin{array}{l}\% \text { within The influence of social agricultural } \\
\text { cooperatives to local development }\end{array}$ & $100,0 \%$ & $100,0 \%$ & $100,0 \%$ & $100,0 \%$ & $100,0 \%$ & $100,0 \%$ \\
\hline
\end{tabular}

Source: Authors' own research

Chi-Square Tests

\begin{tabular}{|c|c|c|c|}
\hline & Value & df & Asymp. Sig. (2-sided) \\
\hline Pearson Chi-Square & $250,643^{\mathrm{a}}$ & 12 &, 000 \\
Likelihood Ratio & 266,969 & 12 &, 000 \\
Linear-by-Linear Association & 159,622 & 1 &, 000 \\
N of Valid Cases & 314 & & \\
\hline
\end{tabular}

a. 5 cells $(25,0 \%)$ have expected count less than 5 . The minimum expected count is, 29 .

The $\mathrm{Pv}$ asymptotic significance value $(0.0001)$ is smaller than 0.05 and the Pearson ChiSquare value (250.643) is bigger than the $\mathrm{df}$ value (21.026), fact that indicates a very 
strong connection between the two analysed variables.

As far as the expectations of the respondents connected with the development of social agricultural cooperatives are concerned, 175 of them $(55.73 \%)$ would prefer grants, 98 $(31.21 \%)$ would prefer reduced taxation and $41(13.06 \%)$ legislative stability.

We must emphasize the fact that most European Union countries support the creation and development of social economic entities by means of support policies, which fight for grants and reduced taxation.

For the question related to the adopted human resources management policy regarding the socially vulnerable people employment availability, $55.5 \%$ of the respondents chose the answer - to a great extent. From the interpretation of the collected data, we can draw the conclusion that a great percentage of the respondents $(68.15 \%$ chose the answer to a great extent) accepts the employment of socially vulnerable people.

Also, we may also notice an availability from the part of small farmers to hire socially vulnerable people, but the great majority (55.73\%) expect support measures for the development of some social economic entities. $64.2 \%$ of the respondents consider that the development of social agricultural cooperatives will greatly influence the economic development of local communities.

\section{Conclusions}

Social economy plays an increasingly important role in the financial market, because it doesn't exert the pressure exerted by shareholders in a private company regarding profitability. The organisation of social economy contributes to the adjustment of three major imbalances that exist on the labour market, namely: unemployment, the instability of employment and the social exclusion of the unemployed.

The present study stresses the fact that small farmers from the south-east of Romania are willing to hire socially vulnerable people, but they also expect some support from the part of the state, materialised in grants, a reduction in taxation and legislative stability. In this regard, we consider that the development of social agricultural cooperatives may relieve the pressure exerted on the social security budget by attracting the people who are part of vulnerable categories (long-term unemployed people, beneficiaries of the guaranteed minimum income) in the activities of the social economic entities and this fact may lead to local and regional economy development. Our future research direction will focus on determining the economic impact of the social agricultural cooperative development on the social security budget in the south-east region of Romania.

\section{References}

Arpinte, D., Baboi, A., Cace, S., Tomescu, C., \& Stanescu, I. (2010). Social inclusion policies. Calitatea vietii XIX, 339-364. Preluat de pe http://www.ies.org.ro

Barna, C. (2014). Atlasul economiei sociale. Bucuresti: Institutul de economie sociala.

CIRIEC. (2015). The Social Economy in the European Union. Bruxelles: European Economic and Social Committee.

Commission, E. (2015, 09 10). Social Entreprenuership. http://ec.europa.eu/social_business

Huttmanova, E. (2016). Sustainable Development and Sustainability Management in the European Union Countries. European Journal of Sustainable Development, 475-482. 
Longo, S., Clark, B., Shriver, T., \& Clausen, R. (2015). Sustenability and Environmental Sociologi: Puting the Economy in its Place and Moving Toward an Integrative Socio-Ecology. Sustainability, 437-445.

Research Report Regarding Social Economy in Romania from a Comparative European Perspective. (2015, 09 10). The Ministry of Labour, Family and Social Protection: http://www.mmuncii.ro

Stanescu, S. (2013). Social Economy Research in Romania. Journal of Social Economy, 40-60.

Wrzaszcz, W., \& Zegar, J. (2016). Economic sustenability of agricultural holdings in poland in the context of their environmental impact. European Journal of Sustainable Development, 497-508. 